\title{
Anti-hyperglycaemic drugs that improve cardiovascular outcomes and a model of diabetic cardiomyopathy.
}

Robert M. Bell

Consultant Cardiologist and Senior Lecturer

Hatter Cardiovascular Institute

University College London and University College London Hospitals NHS Trust

67 Chenies Mews

London

WC1E 6HX

Telephone: $\quad+44(0) 2034479888$

Fax: $\quad$ +44(0) 2034479039

Email: $\quad$ rob.bell@ucl.ac.uk 


\section{Abstract}

Recent cardiovascular outcome trials (CVOTs) have transformed the landscape for the management of type 2 diabetes mellitus. In a remarkably short period of time, national and international guidelines have been overhauled to reflect the remarkable cardiovascular benefits of sodium/glucose linked transporter-2 inhibitors (SGLT2i) and glucagon-like peptite-1 receptor analogues (GLP-1RA) in mitigating cardiovascular risk. Both SGLT2i and GLP-1RAs remain second-line to metformin, reflecting the historical importance of this biguanide anti-hyperglycaemic. In this review, these three very different anti-hyperglycaemics are discussed in the light of CVOT data and of the pre-clinical data revealing remarkable pleiotropic signalling effects of these drugs. A model of diabetic cardiomyopathy is discussed, and the points of contact that these therapeutic interventions have upon this model may of help in understanding how each can be best targeted in this complex pathophysiological disease process.

\section{Keywords:}

Type 2 diabetes mellitus, Metformin, SGLT2 inhibitors, GLP-1 receptor agonists, diabetic cardiomyopathy, cardioprotection.

\section{Introduction}

Since the late $19^{\text {th }}$ century, diabetes mellitus has been recognised as an important cardiovascular risk factor in the development of atherosclerosis ${ }^{1,2}$. Because elevated blood sugar is a principle diagnostic feature of this complex disease, unsurprisingly it was hoped that the control of circulating glucose would improve cardiovascular outcomes in diabetic patients. However, the reality of this approach has not been as rewarding as might have been wished with generally neutral, or even adverse cardiovascular outcomes observed. However, cardiovascular outcome trials (CVOTs), mandated by medicine regulators in both the United States and in Europe, have recently revealed two disparate classes of therapies for the use in type 2 diabetes (T2DM) with a significant and unexpected cardiovascular benefit: the sodium-glucose linked transporter (SGLT2) inhibitors and the Glucagonlike Peptite-1 receptor agonists (GLP-1RA). 


\section{Metformin: the bedrock of contemporary diabetic management}

The cornerstone of current T2DM management is the biguanide, metformin, an insulin-sensitizer. In many respects, metformin could be regarded as the prototypical cardio-protective antihyperglycaemic medication. In the UKPDS 34 study $^{3}$, metformin was compared to both the then standard of care (predominantly diet control) and an "intensive blood-glucose control" group in overweight patients with T2DM. This latter drug-control group used either sulphonylureas or insulin to more effectively lower circulating glucose levels. Interestingly, while reductions in blood sugar were not maintained by any of the drug therapy regimens over the duration of 10-year follow-up metformin, nonetheless, significantly improved cardiovascular outcomes. The incident rate of nonfatal and fatal myocardial infarction/sudden cardiac deaths separated from the control group after approximately one year, and by 10 years, patients on metformin had a significant $39 \%$ risk reduction $(p=0.010)$ compared to patients in the control group ${ }^{3}$ (figure 1 ). By contrast, this composite end-point was not significantly improved by "intensive medical therapy" with sulphonylurea or insulin $(p=0.11)^{3}$. Recent meta-analyses (encompassing 40 trails and 1 million patients) suggest that the extent of the cardiovascular benefits of metformin seen in the UKPDS study is greater than that seen in contemporary practice, however the protection appears robust and significant: comparison of metformin versus non-metformin revealed a significant reduction of cardiovascular mortality with an adjust hazard ratio of 0.81 (95\% confidence interval of $0.79-0.84, p<0.00001){ }^{4}$

The mechanism of this cardioprotective benefit has been extensively studied, with a well-supported literature demonstrating the pleotropic effects that metformin has upon cell survival signalling (for example, $5^{\prime}$ adenosine monophosphate-activated protein kinase $\left(\mathrm{AMPK}^{5}\right)$ and endothelial nitric oxide synthase (eNOS) activation, see review ${ }^{6}$ ).

Metformin has thus been shown to have direct cardioprotective properties and, in historical and contemporary practice, to attenuate cardiovascular mortality.

\section{SGLT2 inhibitors (SGLT2i)}

The SGLT2i ameliorate hyperglycaemia by inhibiting renal reabsorption of glucose via the renal SGLT2 transporter, which is responsible for $90 \%$ of normal reabsorption in the proximal tubule, thus promoting glucosuria and reduction of circulating glucose. Concomitant with glucosuria is a natriuresis, osmotic diuresis, weight reduction (significant calorific loss) and mild ketosis. Empagliflozin, in the EMPA-REG OUTCOME study ${ }^{7}$, revealed a significant cardiovascular outcome benefit in patients with high-risk and established cardiovascular disease. Particularly impressive are their ability to reduce hospital admissions with heart failure, an observation that has been also been 
seen in other drugs within this class, canagliflozin and dapagliflozin (CANVAS ${ }^{8}$ and DECLARE-TIMI $58^{9}$ ) and most recently confirmed in the prospective dapagliflozin heart failure study (DAPA-HF) ${ }^{10}$. What is particularly remarkable regarding these therapies is the rapidity of the divergence of the survival curves - evident within just weeks of initiation of therapy. Thus, cardiovascular outcome benefits are unlikely to be related to attenuation of arterial atherosclerosis. Moreover, there is no difference in the rates of myocardial infarction during the relative short duration of follow up of these investigations. The cardiovascular mortality benefit therefore appears to be through benefits derived through heart failure and perhaps also improved survival from myocardial ischaemia.

The mechanism of the cardioprotection stemming from SGLT2 inhibition has been the subject of intense research, and currently remain unclear. The reduction in the rates of hospitalisation for heart failure way well have its foundation upon the diuretic and natriuretic effects of these drugs with concomitant benefits upon ventricular pre- and after-load. However, the evolution of the diabetic cardiomyopathy (DMCM) through left ventricular hypertrophy and heart failure with preserved ejection fraction (HFpEF) and concluding with a dilated cardiomyopathy and heart failure with reduced ejection fraction (HFrEF) (figure 2 ) is a complex pathophysiological process, ${ }^{11,12}$ one in which SGLT2i may interact. Indeed, SGLT2i appear to have a positive impact upon left ventricular mass in the earlier phases of DMCM and improved ventricular performance (reviewed $\left.{ }^{13}\right)$. The ventricular remodelling and progression from HFpEF to HFrEF appears to be characterised by ischaemic heart disease and ischaemia/reperfusion injury ${ }^{11,12}$, and this too represents a target for SGLT2i.

EMPA-REG, CANVAS and DECLARE were not studies designed to look at myocardial infarction (the number of fatal myocardial infarcts were very low, even within the high-risk population recruited into EMPA-REG). However pre-clinical studies have revealed that SGLT2 inhibition does significantly reduce myocardial infarct size ${ }^{14-16}$. This is surprising: SGLT2 is not widely expressed in man, and there is negligible SGLT2 expression in the heart ${ }^{17}$. Remarkably, SGLT2i cardioprotection is independent of diabetic or glycaemic status, and mediated through cyto-protective signalling: explanted hearts remain protected when perfused, ex-vivo, following oral SGLTi administration ${ }^{14}$. This memory effect may be through the recruitment of cell-survival kinases, through Jak-STAT ${ }^{15}$ or AMPK signalling ${ }^{16}$ and the bolstering of cellular anti-oxidant defences ${ }^{16}$. In contrast, cardioprotection against heart failure may be mediated through preferential metabolism of $\beta$-hydroxybutyrate (augmented by SGLT2i therapy $^{18}$ or through sodium/calcium exchange inhibition ${ }^{19}$, to attenuate adverse calcium accumulation.

With emerging evidence to suggest that SGLT2i may have benefits in all patients, irrespective of diabetic status ${ }^{10}$, the restriction of SGLT2i to patients with diabetes may change. DAPA-HF is the first heart failure study to demonstrate that SGLT2i is equally beneficial in both diabetic and non-diabetic 
patients and this study will be joined by a number of heart failure trials that are due to report in the near future, including the EMPEROR-Reduced and EMEROR-Preserved studies ${ }^{20}$.

\section{Glucagon-like peptide-1 receptor agonists (GLP-1RA)}

Synthetic analogues of the incretin hormone, GLP-1, GLP-1RAs are predominantly injectable antihyperglycaemics, although oral formulations of this class of drug are now emerging. GLP-1RAs stimulate insulin release from pancreatic islet cells to regulate glucose concentration, reduce glucagon secretion, alter gut motility, satiety, lipid metabolism, and thus can lead to reduction of body weight ${ }^{21}$. These properties alone should have a positive impact upon the cardiovascular risk profile, but their benefit is likely to extend beyond their impact upon their canonical role.

The GLP-1RAs are either GLP-1- or Exendin-based (with modification to promote half-life), and it is interesting to note that GLP-1RAs based upon native GLP-1 (liraglutide, semaglutide and albiglutide) have an advantageous impact upon cardiovascular outcomes. LEADER ${ }^{22}$, SUSTAIN- $6^{23}$ and HARMONY ${ }^{24}$ respectively revealed significant reductions in MACCE in patient populations at high risk of developing cardiovascular complications. In contrast to the SGLT2 inhibitors, the time to separation of the survival curves was longer and there was no positive signal in terms of heart failure (non-inferior to placebo); indeed the benefits of GLP-1RAs appear to mediated by the reduction of atherosclerotic-related cardiovascular events (myocardial infarction and stroke) ${ }^{25}$.

Unlike SGLT2, GLP-1 receptors are widely expressed in man, including the heart ${ }^{26}$. Exogenously administered GLP-1 reduces infarct size in rodent models through cytoprotective signalling, involving post-receptor CAMP and CGMP activation ${ }^{27}$, Akt, Erk1/2, p70S6K, and AMPK signalling and inhibition of pro-apoptotic signalling ${ }^{28,29}$. The atherosclerosis benefits of these drugs may be mediated through a beneficial impact upon lipid profiles ${ }^{30}$ and augmented endothelial nitric oxide ${ }^{31}$.

Thus the characteristics of GLP-1-based receptor agonists are different from that seen with SGLT2i, which raises the intriguing possibility that the two classes of therapy may have additive benefit particularly in patients with established coronary or cardiovascular disease.

\section{An emerging paradigm of cardiovascular disease management in diabetes}

Cardioprotection in T2DM has many facets, but evolution and progression of DMCM is a central feature (figure 2). In the model presented, almost any intervention designed to mitigate hyperglycaemia should slow the progression of HFpEF that characterises early and intermediate phases of DMCM. Drugs with pleiotropic effects upon pro-survival cell signalling (e.g. metformin, SGLT2i, GLP-1-based GLP-1RAs) may help prevent progression into late phase DMCM, HFrEF, through attenuating apoptotic myocyte loss and abrogating ischaemia/reperfusion injury from ischaemic heart 
disease. Moreover, GLP-1RAs may have help ameliorate atherosclerotic progression to reduce ischaemic events whereas SGLT2i may help off-load and diurese, thus mitigating excess pre- and afterload and injurious cardiac decompensation. This model may be helpful in developing new therapeutic strategies in diabetes and supports triple therapy with metformin, SGLT2i and GLP-1RA a combination that deserves further investigation.

In summary therefore, building on the foundations of metformin therapy, there are two new classes of glucose-controlling therapies, SGLT2i and GLP-1RA, that each possess remarkable cardiovascular benefits. SGLT2i are clearly beneficial in mitigating heart failure, whereas GLP-1RA may help attenuate atherosclerosis. In clinical practice, the combination of these therapies that appear to have disparate targets may provide the optimum outcomes for diabetic and potentially also for non-diabetic patients, an effect that may have little to do with glucose levels, but through metabolic modulation and recruitment of cellular signalling to promote cellular survival.

\section{Funding and conflict of Interest statement}

RMB receives funding from the British Heart Foundation (Project Grant PG/18/10/33550) and is supported by the National Institute of Health Research University College London Biomedical Research Centre.

$\mathrm{RMB}$ has been in receipt of lecturing honorarium from Merck. There are no conflicts of interest to declare.

\section{Figure legends}

Figure 1 Positive cardiovascular impact of metformin monotherapy upon myocardial infarction (a composite of non-fatal and fatal and sudden death) in the UKPDS 34 study. Compared to diet control (standard therapy), metformin led to a significant reduction of myocardial infarction, whereas there was no significant benefit with intensive therapy with either sulphonylureas and/or insulin, after median 10 year follow up. Figure adapted from UKPDS $34^{3}$.

Figure 2 A proposed model of diabetic cardiomyopathy. Diabetes and its consequences of hyperinsulinaemia and insulin resistance leads to the initial insult upon the cardiovascular system. This initiates a self-sustaining loop of injury, characterised by vascular injury, hypertension, increased afterload and myocardial hypertrophy and subsequent diastolic impairment and myocardial fibrosis. As this becomes symptomatic, these features characterise heart failure with preserved ejection fraction (HFpEF). Overtime, the 
sustained strain and injury upon the left ventricle leads to myocyte loss through apoptotic cell death, which, in combination with the development of coronary atherosclerosis and ischaemic heart disease and the initiation of a second self-sustaining loop of Heart failure with reduced ejection fraction (HFrEF) and the characteristic late-phase dilated cardiomyopathy.

\section{References}

1. P. V. De l'angine de poitrine dans ses rapports avec le diabete. Gaz hedb de ned (ser 2) 1883;20:364.

2. $\quad$ B. N. Der Diabetes Mellitus. . Vienna: Holder 1906;2nd edn. :260.

3. Effect of intensive blood-glucose control with metformin on complications in overweight patients with type 2 diabetes (UKPDS 34). UK Prospective Diabetes Study (UKPDS) Group. Lancet 1998;352:854-865.

4. Han Y, Xie H, Liu Y, Gao P, Yang X, Shen Z. Effect of metformin on all-cause and cardiovascular mortality in patients with coronary artery diseases: a systematic review and an updated meta-analysis. Cardiovasc Diabetol 2019;18:96.

5. Whittington HJ, Hall AR, McLaughlin CP, Hausenloy DJ, Yellon DM, Mocanu MM. Chronic metformin associated cardioprotection against infarction: not just a glucose lowering phenomenon. Cardiovasc Drugs Ther 2013;27:5-16.

6. Varjabedian L, Bourji M, Pourafkari L, Nader ND. Cardioprotection by Metformin: Beneficial Effects Beyond Glucose Reduction. Am J Cardiovasc Drugs 2018;18:181-193.

7. Zinman B, Wanner C, Lachin JM, Fitchett D, Bluhmki E, Hantel S, Mattheus M, Devins T, Johansen OE, Woerle HJ, BroedI UC, Inzucchi SE. Empagliflozin, Cardiovascular Outcomes, and Mortality in Type 2 Diabetes. New Eng/ J Med 2015;373:2117-2128.

8. Neal B, Perkovic V, Mahaffey KW, de Zeeuw D, Fulcher G, Erondu N, Shaw W, Law G, Desai M, Matthews DR, Group CPC. Canagliflozin and Cardiovascular and Renal Events in Type 2 Diabetes. N Engl J Med 2017;377:644-657.

9. Wiviott SD, Raz I, Bonaca MP, Mosenzon O, Kato ET, Cahn A, Silverman MG, Zelniker TA, Kuder JF, Murphy SA, Bhatt DL, Leiter LA, McGuire DK, Wilding JPH, Ruff CT, Gause-Nilsson IAM, Fredriksson M, Johansson PA, Langkilde AM, Sabatine MS, Investigators D-T. Dapagliflozin and Cardiovascular Outcomes in Type 2 Diabetes. N Engl J Med 2019;380:347357.

10. McMurray JJV, Solomon SD, Inzucchi SE, Kober L, Kosiborod MN, Martinez FA, Ponikowski P, Sabatine MS, Anand IS, Belohlavek J, Bohm M, Chiang CE, Chopra VK, de Boer RA, Desai AS, Diez M, Drozdz J, Dukat A, Ge J, Howlett JG, Katova T, Kitakaze M, Ljungman CEA, Merkely B, Nicolau JC, O'Meara E, Petrie MC, Vinh PN, Schou M, Tereshchenko S, Verma S, Held C, DeMets DL, Docherty KF, Jhund PS, Bengtsson O, Sjostrand M, Langkilde AM, Committees DHT, Investigators. Dapagliflozin in Patients with Heart Failure and Reduced Ejection Fraction. N Engl J Med 2019.

11. Lee MMY, McMurray JJV, Lorenzo-Almoros A, Kristensen SL, Sattar N, Jhund PS, Petrie MC. Diabetic cardiomyopathy. Heart 2018.

12. Jia G, Hill MA, Sowers JR. Diabetic Cardiomyopathy: An Update of Mechanisms Contributing to This Clinical Entity. Circ Res 2018;122:624-638.

13. Lan NSR, Fegan PG, Yeap BB, Dwivedi G. The effects of sodium-glucose cotransporter 2 inhibitors on left ventricular function: current evidence and future directions. ESC Heart Fail 2019. 
14. Lim VG, Bell RM, Arjun S, Kolatsi-Joannou M, Long DA, Yellon DM. SGLT2 Inhibitor, Canagliflozin, Attenuates Myocardial Infarction in the Diabetic and Nondiabetic Heart. JACC Basic Transl Sci 2019;4:15-26.

15. Andreadou I, Efentakis $P$, Balafas $E$, Togliatto $G$, Davos $C H$, Varela A, Dimitriou CA, Nikolaou PE, Maratou E, Lambadiari V, Ikonomidis I, Kostomitsopoulos N, Brizzi MF, Dimitriadis G, Iliodromitis EK. Empagliflozin Limits Myocardial Infarction in Vivo and Cell Death in Vitro: Role of STAT3, Mitochondria, and Redox Aspects. Front Physiol 2017;8:1077.

16. Sayour AA, Korkmaz-Icoz S, Loganathan S, Ruppert M, Sayour VN, Olah A, Benke K, Brune M, Benko R, Horvath EM, Karck M, Merkely B, Radovits T, Szabo G. Acute canagliflozin treatment protects against in vivo myocardial ischemia-reperfusion injury in non-diabetic male rats and enhances endothelium-dependent vasorelaxation. J Transl Med 2019;17:127.

17. Chen J, Williams S, Ho S, Loraine H, Hagan D, Whaley JM, Feder JN. Quantitative PCR tissue expression profiling of the human SGLT2 gene and related family members. Diabetes Ther 2010;1:57-92.

18. Ferrannini E, Mark M, Mayoux E. CV Protection in the EMPA-REG OUTCOME Trial: A "Thrifty Substrate" Hypothesis. Diabetes Care 2016;39:1108-1114.

19. Uthman L, Baartscheer A, Schumacher CA, Fiolet JWT, Kuschma MC, Hollmann MW, Coronel R, Weber NC, Zuurbier CJ. Direct Cardiac Actions of Sodium Glucose Cotransporter 2 Inhibitors Target Pathogenic Mechanisms Underlying Heart Failure in Diabetic Patients. Front Physiol 2018;9:1575.

20. Savarese G, Sattar N, Januzzi J, Verma S, Lund LH, Fitchett D, Zeller C, George JT, Brueckmann M, Ofstad AP, Inzucchi SE, Wanner C, Zinman B, Butler J. Empagliflozin Is Associated With a Lower Risk of Post-Acute Heart Failure Rehospitalization and Mortality. Circulation 2019;139:1458-1460.

21. Shah M, Vella A. Effects of GLP-1 on appetite and weight. Rev Endocr Metab Disord 2014;15:181-187.

22. Marso SP, Daniels GH, Brown-Frandsen K, Kristensen P, Mann JF, Nauck MA, Nissen SE, Pocock S, Poulter NR, Ravn LS, Steinberg WM, Stockner M, Zinman B, Bergenstal RM, Buse JB, Committee LS, Investigators LT. Liraglutide and Cardiovascular Outcomes in Type 2 Diabetes. N Engl J Med 2016;375:311-322.

23. Marso SP, Bain SC, Consoli A, Eliaschewitz FG, Jodar E, Leiter LA, Lingvay I, Rosenstock J, Seufert J, Warren ML, Woo V, Hansen O, Holst AG, Pettersson J, Vilsboll T, Investigators S-. Semaglutide and Cardiovascular Outcomes in Patients with Type 2 Diabetes. N Engl J Med 2016;375:1834-1844.

24. Hernandez AF, Green JB, Janmohamed S, D'Agostino RB, Sr., Granger CB, Jones NP, Leiter LA, Rosenberg AE, Sigmon KN, Somerville MC, Thorpe KM, McMurray JJV, Del Prato S, Harmony Outcomes c, investigators. Albiglutide and cardiovascular outcomes in patients with type 2 diabetes and cardiovascular disease (Harmony Outcomes): a double-blind, randomised placebo-controlled trial. Lancet 2018;392:1519-1529.

25. Tanaka A, Node K. Clinical application of glucagon-like peptide-1 receptor agonists in cardiovascular disease: lessons from recent clinical cardiovascular outcomes trials. Cardiovasc Diabetol 2018;17:85.

26. Baggio LL, Yusta B, Mulvihill EE, Cao X, Streutker CJ, Butany J, Cappola TP, Margulies KB, Drucker DJ. GLP-1 Receptor Expression Within the Human Heart. Endocrinology 2018;159:1570-1584.

27. Ban K, Noyan-Ashraf MH, Hoefer J, Bolz SS, Drucker DJ, Husain M. Cardioprotective and vasodilatory actions of glucagon-like peptide 1 receptor are mediated through both glucagon-like peptide 1 receptor-dependent and -independent pathways. Circulation 2008;117:2340-2350.

28. Bose AK, Mocanu MM, Carr RD, Brand CL, Yellon DM. Glucagon-like peptide 1 can directly protect the heart against ischemia/reperfusion injury. Diabetes 2005;54:146-151. 
29. Bose AK, Mocanu MM, Carr RD, Yellon DM. Myocardial ischaemia-reperfusion injury is attenuated by intact glucagon like peptide-1 (GLP-1) in the in vitro rat heart and may involve the p70s6K pathway. Cardiovasc Drugs Ther 2007;21:253-256.

30. Anholm C, Kumarathurai P, Pedersen LR, Samkani A, Walzem RL, Nielsen OW, Kristiansen OP, Fenger M, Madsbad S, Sajadieh A, Haugaard SB. Liraglutide in combination with metformin may improve the atherogenic lipid profile and decrease C-reactive protein level in statin treated obese patients with coronary artery disease and newly diagnosed type 2 diabetes: A randomized trial. Atherosclerosis 2019;288:60-66.

31. Barale C, Buracco S, Cavalot F, Frascaroli C, Guerrasio A, Russo I. Glucagon-like peptide 1related peptides increase nitric oxide effects to reduce platelet activation. Thromb Haemost 2017;117:1115-1128. 


\section{UKPDS-34: Myocardial infarction}

(composite of non-fatal and fatal MI and sudden death)

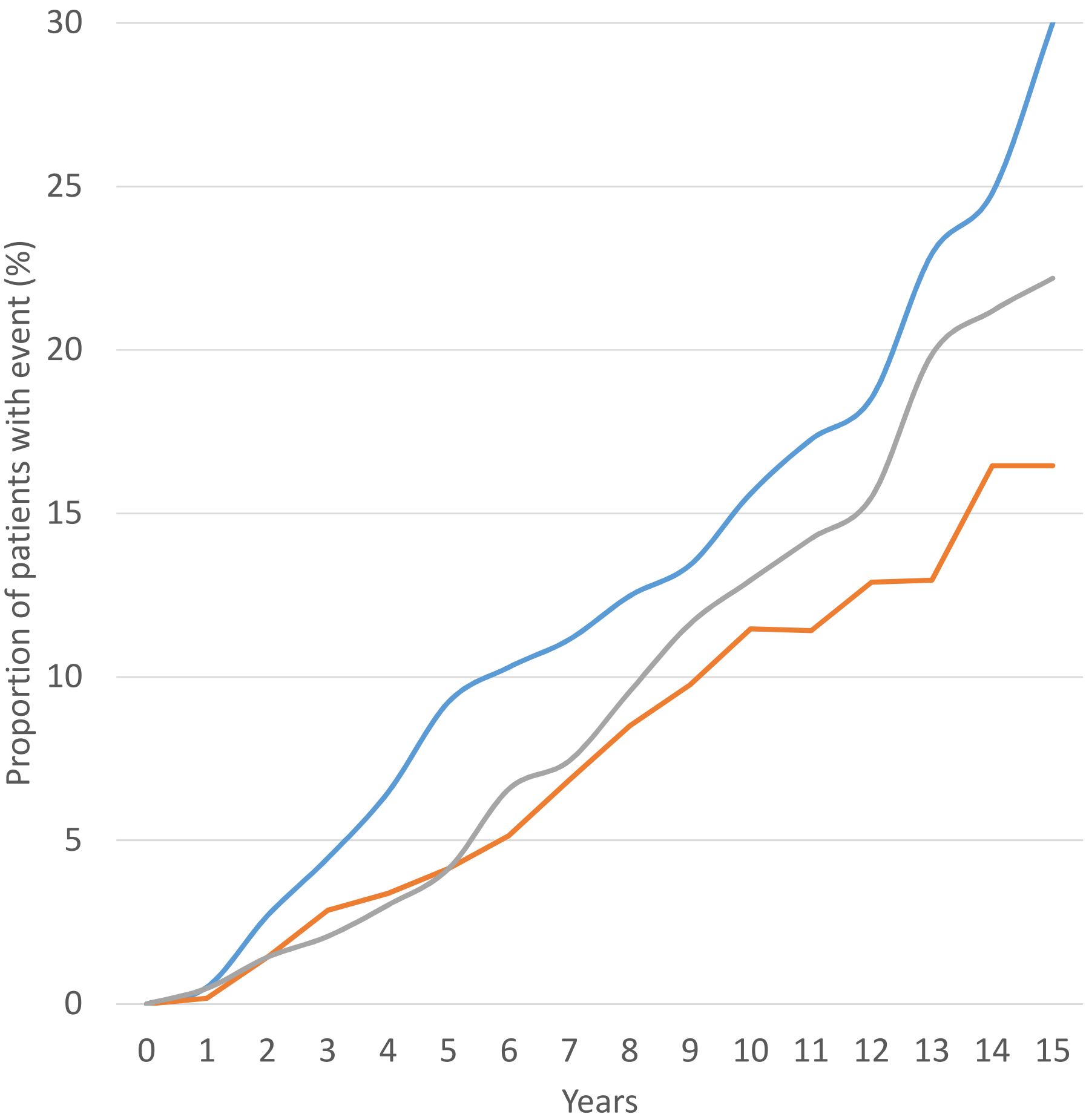

—Standard therapy —Metformin —Intensive 


\section{Diabetes AGEs, glucotoxicity}

hyperglycaemia Insulinaemia Insulin resistance

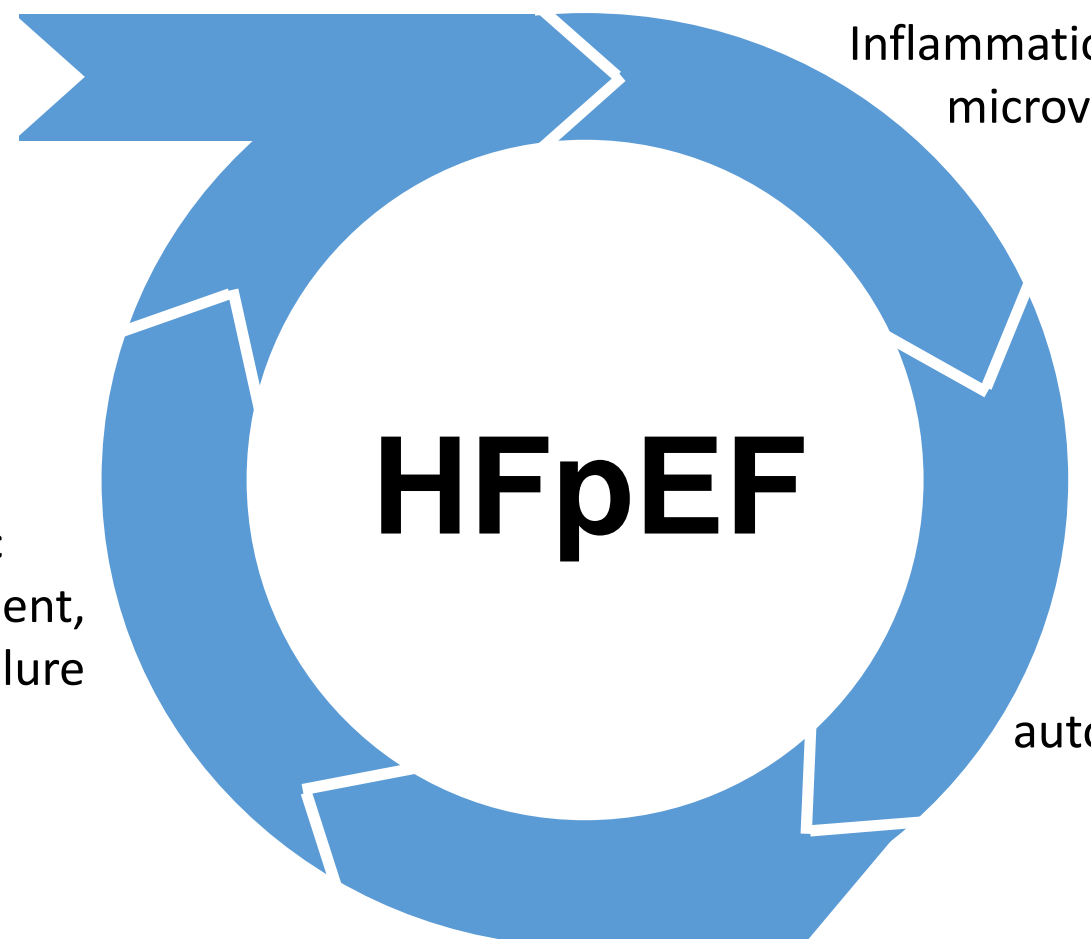

Vascular stiffness,

Diastolic impairment, heart failure

Myocardial hypertrophy, fibrosis, myocardial stiffening, apoptosis

Myocyte apoptosis, Coronary artery disease and myocardial infarction activation of RAAS, autonomic dysfunction

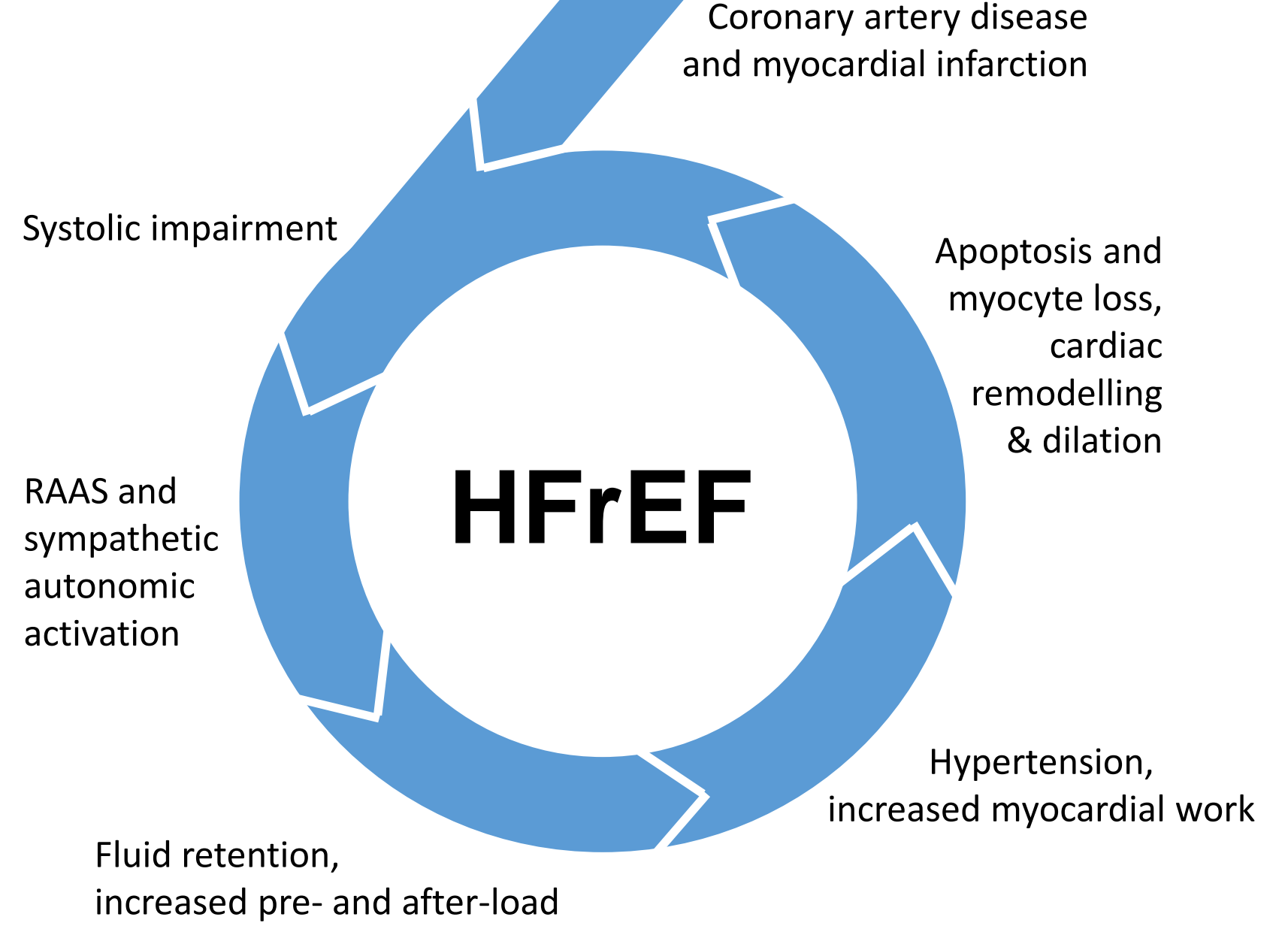

